\title{
ANDES

www.scielo.cl

\section{Recomendaciones de organización, diseño, características y funcionamiento de servicios o unidades de neonatología}

\author{
Recommendations on organization, design, characteristics and operation \\ of neonatology services
}

\section{Galo Bajaña R. ${ }^{a, j}$, Fernando Carvajal E. ${ }^{b, j}$, Javier Cifuentes R. ${ }^{c, j}$, Pamela Gallardo C. ${ }^{\mathrm{d}, j}$, Javier Kattan S. ${ }^{\mathrm{ej}}$, Álvaro Méndez F. ${ }^{\mathrm{f} j}$, José Antonio Salinas T.g,j, Daniela Sandino P. ${ }^{\mathrm{a}, \mathrm{h}, \mathrm{j}}$, Soledad Urzúa B. ${ }^{\mathrm{e}, \mathrm{i}, \mathrm{j}}$}

\begin{abstract}
aHospital Dr. Gustavo Fricke. Viña del Mar, Chile
'Clínica Indisa. Escuela de Medicina, Universidad Andrés Bello. Santiago, Chile

dEnfermera Matrona

eEscuela de Medicina, Pontificia Universidad Católica de Chile. Santiago, Chile

${ }^{\text {fH}}$ Hospital Dr. César Garavagno Burotto de Talca. Talca, Chile

${ }^{9}$ Hospital San Juan de Dios. Clínica las Condes. Santiago, Chile

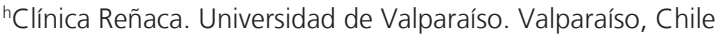

'Rama de Neonatología Sociedad Chilena de Pediatría Chile. Santiago, Chile

Recibido: 01 de diciembre de 2020; Aceptado: 27 de diciembre de 2020

¿Qué se sabe del tema que trata este estudio?

La organización materno-neonatal en redes de atención con unidades de distinto nivel de complejidad atendiendo a la embarazada según el nivel de riesgo materno-fetal ha demostrado ser una de las medidas de mayor impacto en la morbimortalidad maternoneonatal.
\end{abstract}

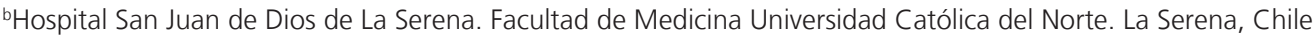

¡Grupo Asesor de Neonatología, División de Gestión de Redes Asistenciales (DIGERA), Subsecretaría de Redes Asistenciales. Ministerio de Salud de

\section{¿Qué aporta este estudio a lo ya conocido?}

Se actualizan las recomendaciones nacionales para la organización y diseño de la atención neonatal pública y privada, manteniendo un modelo de atención en red y recomendando un diseño e infraestructura modernos concordantes con una atención centrada en la familia.

\section{Resumen}

La atención neonatal ha experimentado importantes avances que involucran tecnología para el tratamiento y monitorización, diseño de los espacios de atención, incorporación de profesionales de apoyo y especialmente el fortalecimiento de un modelo de organización en redes con centros de distintos niveles de complejidad. Las unidades neonatales deben ubicarse en instituciones con servicios de maternidad e idealmente con servicios de pediatría de complejidad equivalentes. Este documento define criterios de admisión según el nivel de complejidad y criterios de traslado entre los diferentes niveles.
Palabras clave:

Recién Nacido;

Neonatología;

Niveles de Atención de Salud;

Unidades de Intensivo

Neonatal;

Enfermería Neonatal;

Recursos Humanos

Correspondencia:

Javier Cifuentes $\mathrm{R}$.

jcifuentes@neored.net 
La evidencia recomienda un diseño de pieza individual por los beneficios asociados: disminución de ocurrencia y mejor control de infecciones asociadas a la atención de salud, favorecer la lactancia y la interacción con los padres. Los tamaños sugeridos favorecen la implementación del modelo de atención centrado en la familia. Estas recomendaciones establecen la posibilidad de realizar procedimientos quirúrgicos de urgencia en la unidad neonatal y los requerimientos de seguridad de la planta física. Se define la dotación de recursos humanos según nivel de complejidad reconociendo el tiempo dedicado a actividades no asistenciales, pero necesarias para la gestión. La atención neonatal debe ser liderada por el neonatólogo como subespecialista, permitiendo la participación de pediatras generales con formación y experiencia demostrada en la atención neonatal. Las matronas o enfermeras deben contar con capacitación certificada en neonatología. Además, se definen requerimientos de profesionales de apoyo tales como, nutricionistas, psicólogos, kinesiólogos, terapistas ocupacionales, fonoaudiólogos químico-farmacéuticos y asistentes sociales. El objetivo de este documento es actualizar las "Recomendaciones sobre Organización, Características y Funcionamiento de Servicios o Unidades de Neonatología” para que sirvan como orientación y guía para el diseño y la gestión neonatal de los centros de atención de salud pública y privada del país.

\begin{abstract}
Neonatal care has undergone important advances involving the technology for treatment and monitoring, the design of care spaces, the incorporation of support professionals, and, especially, the strengthening of an organizational model in networks with centers of different levels of care. Neonatal units should be located in centers with maternity services and, ideally, with pediatric ones of an equivalent level of care. This document defines the admission and transfer criteria according to the level of care and among the different levels, respectively. The evidence recommends an individual room design due to the associated benefits such as decreased occurrence and better control of healthcare-associated infections, improved breastfeeding, and better interaction with parents. The suggested room sizes favor the implementation of the family-centered care model. These recommendations establish the possibility of performing emergency surgical procedures in the neonatal unit and define the safety criteria of the physical plant. In addition, they define the human resources according to the level of care, recognizing the time dedicated to non-direct patient care activities, , and the requirements of non-medical professionals such as psychologists, physical and respiratory therapists, occupational therapists, speech therapists, pharmacists, dietitians, and social workers. Neonatal care should be led by the neonatologist allowing the participation of general pediatricians with training and demonstrated experience in neonatal care. Midwives and neonatal nurses should have accredited formation in such area. The purpose of this document is to update the "Recommendations on the Organization, Characteristics and Operation of Neonatology Services or Units" to serve as an orientation and guide for the design and management of neonatal care in public and private health care centers in the country.
\end{abstract}

\section{Keywords:}

Infant;

Newborn;

Neonatology Health

Care Levels;

Intensive Care Units;

Neonatal;

Neonatal Nursing;

Human Resources

\section{Introducción}

Las experiencias del modelo de atención perinatal desarrollado en distintos países del mundo han demostrado que para mejorar las cifras de mortalidad neonatal es importante organizar el cuidado perinatal de modo que se pueda entregar el mejor tratamiento y de la manera más oportuna a cada paciente, lo que es particularmente difícil en países de geografía como la nuestra.

Una de las estrategias implementadas ha sido definir distintos niveles de cuidado obstétrico y neona$\mathrm{tal}^{1-4}$, los que tratan a embarazadas y recién nacidos de acuerdo con el riesgo, idealmente definido antes de nacer, concentrando los niños de mayor riesgo y aquellos que presentan patologías graves en grandes centros re- gionales. Este tipo de centros corresponden a unidades de referencia para una o varias redes y concentran la atención de patologías médicas o quirúrgicas, que por su baja frecuencia de presentación deben ser atendidos por equipos especializados con gran experiencia y apoyados por equipamiento diagnóstico y terapéutico avanzados. La evidencia muestra mejores resultados obstétricos y neonatales cuando los centros concentran un mayor volumen de pacientes producto de la experiencia acumulada por los equipos clínicos ${ }^{5-17}$.

El modelo de organización en red para la atención perinatal tiene entre sus objetivos prevenir el traslado neonatal, privilegiando el traslado materno cuando por riesgo materno o fetal corresponda ${ }^{8,9,18}$. Si bien la mayoría de los $\mathrm{RN}$ de alto riesgo que requieren cuidados especiales pueden identificarse prenatalmente, 
aproximadamente un $25 \%$ de los niños que requieren cuidado crítico no tiene factores de riesgo identificados previo al nacimiento. Por este motivo, todos los niveles de atención perinatales deben estar capacitados para diagnosticar situaciones de riesgo y dar la asistencia necesaria para estabilizar al recién nacido mientras se efectúa su traslado al centro de red con la capacidad resolutiva que corresponda ${ }^{4,19}$. Gracias a estas estrategias se han conseguido importantes mejorías en la sobrevi-

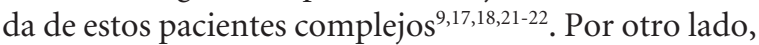
desde el punto de vista económico, el modelo de organización en redes de atención optimiza los recursos al hacer confluir recursos técnicos y humanos en centros que reciben los pacientes que los requieren.

En este contexto, en el modelo de organización de la Red de Alta Complejidad Neonatal, las unidades de hospitalización neonatal se clasifican en 4 niveles de complejidad según su capacidad resolutiva y la del establecimiento en que se encuentran. De este modo los sistemas de atención materno-infantil público y privado deben tender a un modelo en que todo establecimiento que atienda partos cuente con una unidad de hospitalización neonatal cuya complejidad sea concordante con la capacidad resolutiva del servicio de Gineco-obstetricia y de Pediatría con que cuenta. Los distintos niveles de complejidad se encuentran interconectados a través de criterios de referencia y contrarreferencia comunes, sistemas de teleasistencia y de traslado de pacientes.

En cada establecimiento los Centros de Responsabilidad de la Atención Neonatal son los garantes de la tuición técnica de la atención intrahospitalaria del recién nacido en sala de partos, pabellón de maternidad, puerperio y en unidades de hospitalización neonatal o unidades mixtas pediátrico-neonatales y de la supervisión de salud ambulatoria de los recién nacidos de riesgo egresados de las unidades de hospitalización. Bajo la tuición técnica del centro de responsabilidad neonatal, el establecimiento puede mantener en área de puerperio pacientes neonatales cuyo nivel de cuidado requerido corresponda a cuidados básicos. Los pacientes que requieran cuidados medios deben hospitalizarse en unidades neonatales o unidades pediátrico-neonatales, según sea la organización del centro de salud correspondiente.

En base a la información reportada en la literatura sobre los estándares de atención neonatal ${ }^{2,23-26}$, a las recomendaciones realizadas por la Rama de Neonatología de la Sociedad Chilena de Pediatría en el año $2009^{27}$, las recomendaciones del Ministerio de Salud de Chile ${ }^{28,29}$ y a la realidad nacional actual, el grupo de trabajo asesor de neonatología de la Subsecretaría de Redes Asistenciales ha actualizado las "Recomendaciones sobre Organización, Características y Funcionamiento de Servicios o Unidades de Neonatología” para que sir- van como orientación y guía para el diseño y la gestión neonatal de los centros de atención de salud pública y privada del país.

Las recomendaciones propuestas por el grupo de trabajo asesor de neonatología, en línea con las recomendaciones del Ministerio de Salud de Chile para la programación de la atención en red $^{30}$, consideran los distintos aspectos relevantes en la organización neonatal como definición de niveles de complejidad, recursos humanos, equipamiento e infraestructura, entre otros necesarios para cumplir con el objetivo de reducir la morbimortalidad perinatal en un ambiente de atención centrada en la familia, en la seguridad del paciente y del equipo de salud, con un sistema de evaluación de procesos y resultados que permita su monitoreo y la introducción oportuna de los cambios necesarios para su mejora continua.

\section{Definición de los servicios o unidades}

Se define como Servicio o Unidad de Neonatología a la Unidad Funcional que garantiza la cobertura asistencial de los pacientes neonatos enfermos, como también, la asistencia a los recién nacidos en la sala de partos o pabellón y la atención del neonato que se encuentra en puerperio con su madre.

Desde el punto de vista operacional se considera período de atención neonatal desde el momento del nacimiento hasta las 44 semanas de edad post-menstrual, con un mínimo de 28 días y sin restricciones de peso al nacer. Los pacientes hospitalizados mayores de 44 semanas de edad post- menstrual deben ser derivados a Servicios de Pediatría por haber superado el período de atención neonatal y la patología propia del neonato. En algunas patologías agudas del neonato pretérmino tardío o de término (ej. patologías quirúrgicas, neurológicas, etc.), este podría permanecer en el Servicio de Neonatología hasta que la patología en su fase aguda sea superada, con un máximo de 8 semanas de vida.

Se sugiere que las Unidades Funcionales de Neonatología, según su nivel de complejidad, integren dentro de sus responsabilidades los siguientes procesos asistenciales:

- La atención prenatal y perinatal en colaboración con otros especialistas, especialmente obstetras.

- La asistencia al recién nacido en el parto.

- La atención al recién nacido que permanece con su madre en la maternidad.

- La asistencia al recién nacido hospitalizado en sala de cuidados intensivos, cuidados intermedios, cuidados medios o básicos.

- La coordinación para la atención domiciliaria en neonatos derivados con cuidados domiciliarios.

- Colaboración de la atención en consulta de seguimiento del recién nacido de riesgo. 


\section{Clasificación de los servicios o unidades neonatales}

Los Servicios o Unidades de Neonatología deben organizarse considerando un modelo de atención en red que incluya distintos niveles de resolución coordinados entre ellos.

Los distintos niveles de complejidad deberán ajustarse de acuerdo con:

- Capacidad resolutiva.

- Población de referencia: recién nacidos en el mismo centro y toda el área de referencia (según corresponda al nivel de complejidad).

- Capacidad resolutiva de los Servicio de Ginecología y Obstetricia de los Hospitales o Clínicas en los que se encuentre la Unidad Neonatal.

- Accesibilidad en consideración a variables geográficas.

El tipo de recién nacido que se atenderá en cada nivel dependerá de la categorización según riesgo y dependencia (CUDYR) y según riesgo asociado a su diagnóstico específico.

En el diseño de nuevos establecimientos hospitalarios se debe considerar un Servicio de Maternidad solamente cuando el número de partos anuales esperados sea igual o superior a 1000. En casos especiales, en los que, atendiendo a razones históricas, culturales o geográficas, se diseñen centros hospitalarios con un número esperado de atención de partos anuales inferior a 1000, el centro debe considerar en su proyecto una Unidad Neonatal de Nivel I con al menos un cupo de estabilización para el RN que requiera ser trasladado a un establecimiento de mayor complejidad. El cupo de estabilización tendrá las características de cupo UTI, con acceso a VM e incubadora de transporte. Así mismo los establecimientos de baja complejidad actualmente en funcionamiento que atienden partos y que no cuenten con una unidad de Neonatología Nivel I deben contar con una Sala de cuidado básico del RN en Puericultura con un cupo de estabilización para el RN que nace con patología no diagnosticada prenatalmente o que presenta un evento patológico en el período de atención inmediata o puerperio.

\section{Características y requerimientos de las unida- des neonatales}

En la tabla 1 se muestran las características de las unidades neonatales y requisitos mínimos de organización según su nivel de complejidad. Estas recomendaciones para los nuevos proyectos hospitalarios públicos y privados se entienden como el mínimo necesario para proveer una atención neonatal segura y centrada en la familia y deben estar abiertas a modificaciones en la medida que los modelos de atención incorporen nuevos elementos organizacionales y técnicos que impacten en los resultados sanitarios de este grupo etario. Las unidades neonatales actualmente en funcionamiento y con autorización sanitaria vigente podrán en la medida de sus posibilidades ajustar su planta física a algunas de estas recomendaciones.

Los niveles de complejidad están definidos de modo de estructurar un funcionamiento en redes de atención que facilite la concentración de pacientes complejos en centros de experiencia regionales o nacionales. Por su impacto en la mortalidad infantil y neonatal, y por sus requerimientos de cuidados específicos ambulatorios y hospitalarios a lo largo de la vida, se consideran pacientes complejos a los recién nacidos prematuros menores de 32 semanas de edad gestacional y a los recién nacidos con malformaciones mayores. El objetivo de esta propuesta de organización es evitar la atomización de la atención neonatal de estos pacientes en unidades pequeñas con baja carga asistencial, ya que la literatura y los modelos internacionales muestran que los mejores resultados sanitarios se asocian a centros con cargas asistenciales que aseguren su desarrollo como centros de excelencia. La autoridad sanitaria puede autorizar el funcionamiento de Unidades Nivel III con un volumen de partos y/o población de referencia menores a las especificadas en la tabla 1 en situaciones geográficas especiales de aislamiento o distancia a otros centros de salud.

La infraestructura y el equipamiento requerido para cada nivel de atención neonatal están definidos, en general, en la versión vigente del documento Norma Técnica Básica (NTB) del Ministerio de Salud de Chile.

Configuración general: El Servicio o Unidad de Neonatología debe cumplir con los requerimientos mínimos de equipamiento e infraestructura para disminuir riesgo de infecciones nosocomiales, accidentes y permitir el flujo expedito y la estancia de pacientes, equipo de salud y padres. Debe disponerse de espacio para asistencia, bodegaje, equipos, capacitación y espacios administrativos y de gestión ${ }^{23,25,27,31-36}$.

En lo que respecta al diseño de la planta física de las Unidades de Neonatología de nivel de complejidad II, III y IV se recomienda, al igual que para las unidades pediátricas, el modelo de habitación individual, ya que es el que la evidencia muestra como el diseño que mejor se adapta a las necesidades de un modelo de atención centrado en la familia, además de favorecer el neurodesarrollo, disminuir el riesgo de infecciones asociadas a la atención de salud y asegurar el cumplimiento con los estándares de privacidad. Las unidades nivel de complejidad III o IV pueden 


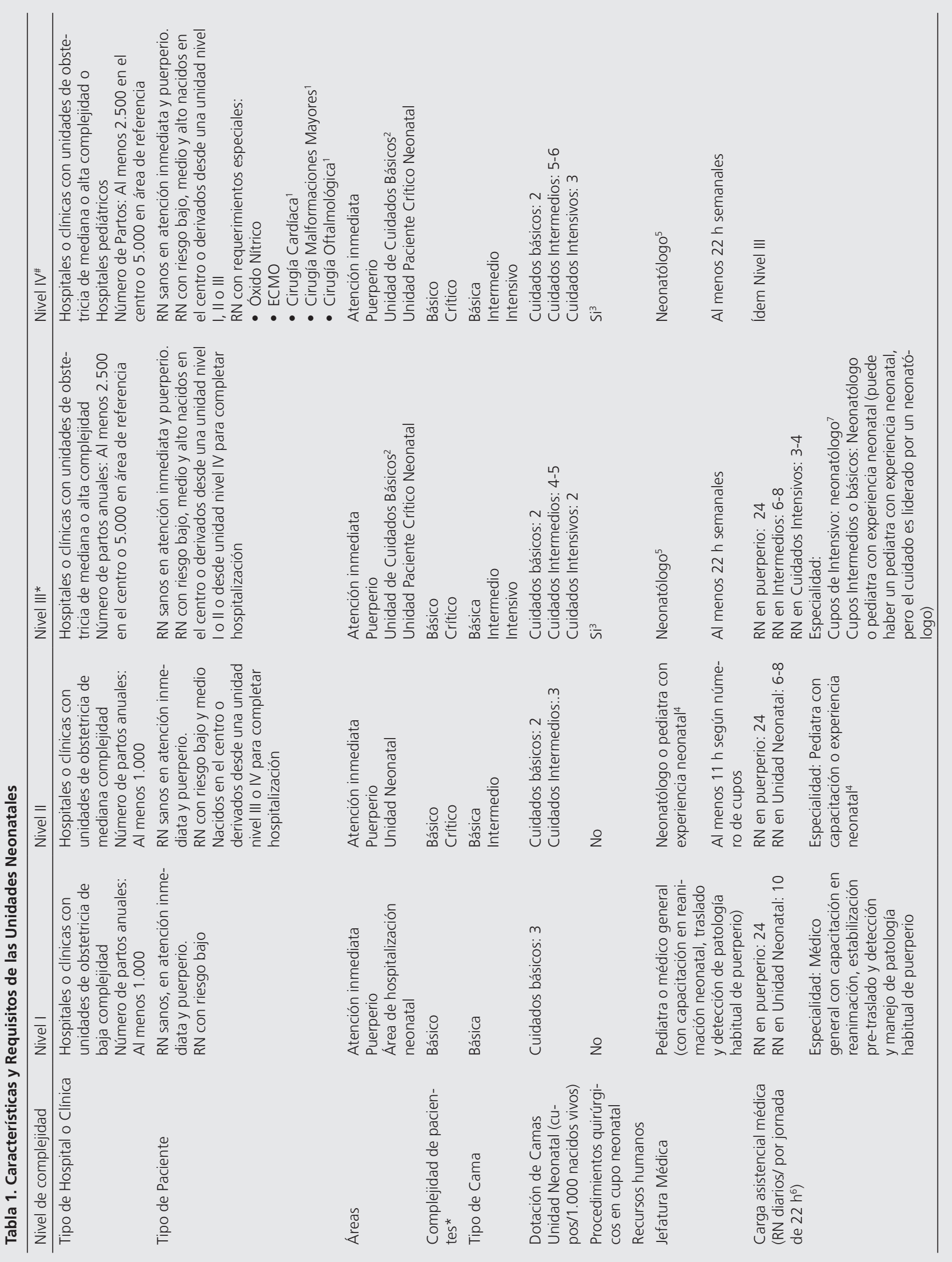




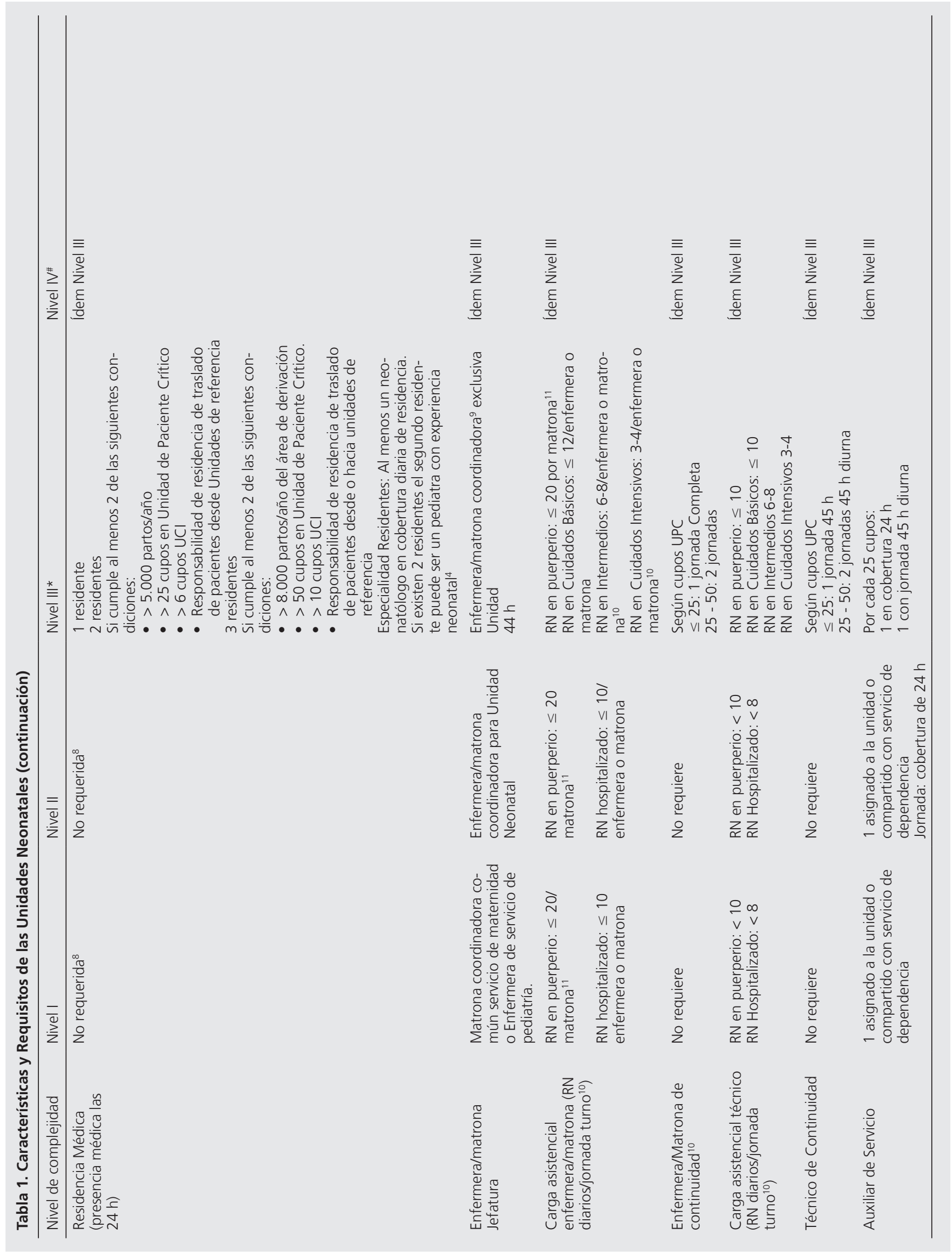




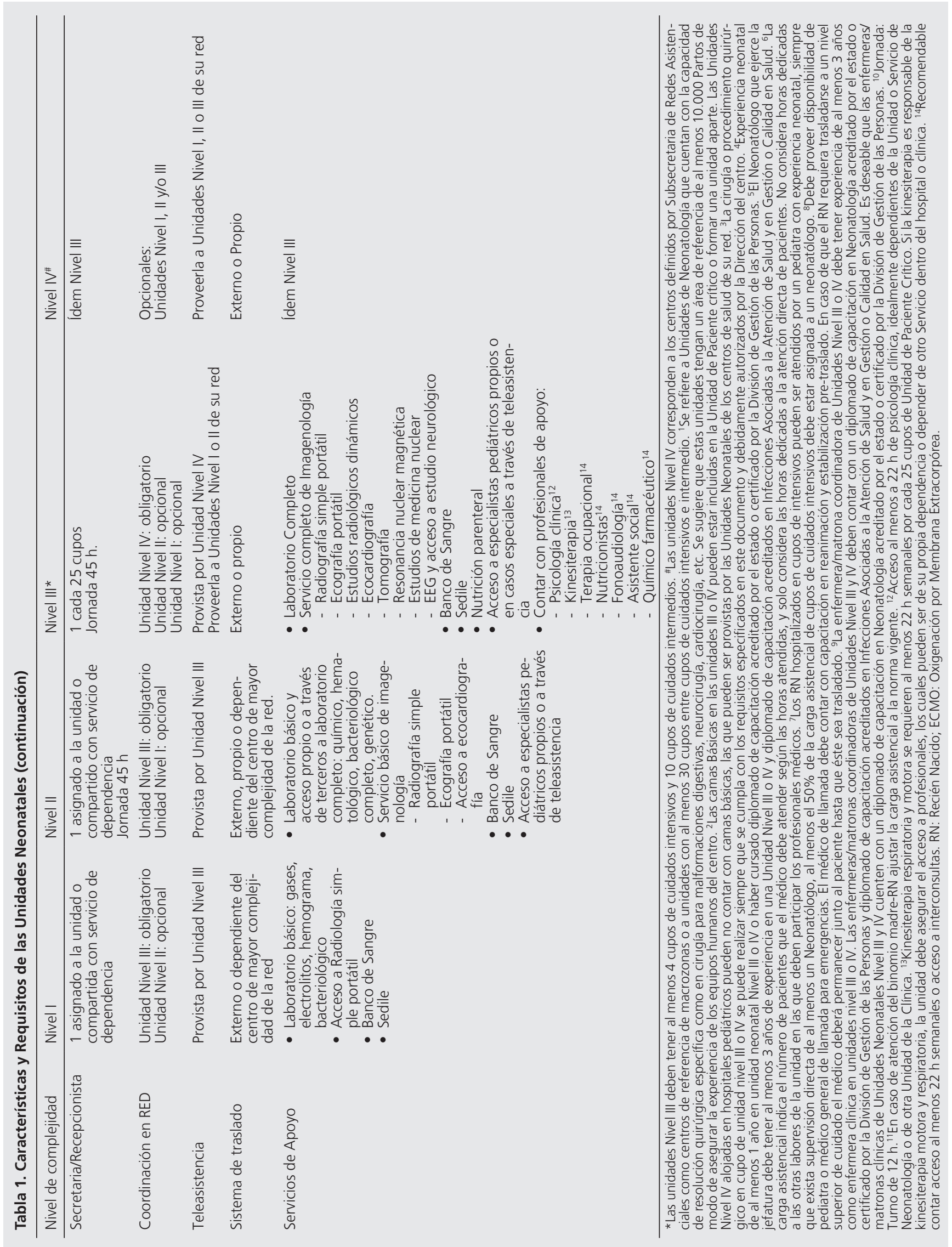


organizar sus cupos de intensivo e intermedio en un modelo de unidad de paciente crítico con cupos indiferenciados o mantener recintos separados para ambos tipos de cupos. La superficie asignada a cada cupo debe tener el espacio suficiente para dar cabida a la cuna o incubadora del recién nacido, a los equipos de monitorización y tratamiento, permitir el desplazamiento de equipos de radiología y ecografía y asegurar suficiente espacio para la permanencia prolongada de la familia con mobiliario cómodo (sillones reclinables) que permitan y faciliten la lactancia, la aplicación de método madre canguro y la interacción de los padres con el recién nacido. En el caso que el modelo de organización sea el de una unidad de paciente crítico indiferenciado, el tamaño de los cupos debe ser equivalente al de un cupo de intensivo.

\section{Localización dentro del hospital}

Debe estar cerca de las salas de parto y pabellones de maternidad y lejos del tránsito de personas de otras unidades del hospital. Para las unidades que reciben traslados desde otros establecimientos, debe asegurarse un acceso expedito desde donde llegan las ambulancias al hospital.

\section{Espacios requeridos}

a) Cupo UCI: La dimensión de un cupo UCI debe ser de un mínimo de 16 a $20 \mathrm{mt}^{2}$. Los cupos UCI deben contar con sistemas de control de temperatura independientes y renovación de aire según lo establecido por departamento de infecciones asociadas a la atención de salud de modo que se cumpla con la seguridad necesaria para la realización de procedimientos invasivos.

b) Cupo UTI: La dimensión de un cupo UTI debe ser de un mínimo de 14 a $16 \mathrm{mt}^{2}$. En el caso que el modelo de organización sea el de una unidad de paciente crítico el tamaño de los cupos debe ser el equivalente al de un cupo de intensivo.

c) Cupo Básico: La dimensión de un cupo básico debe ser de un mínimo de 12 a $14 \mathrm{mt}^{2}$.

d) Cupo de aislamiento: Al menos el 20\% de los cupos de una unidad nivel III o IV deben reunir las características de cupos de aislamiento. Los cupos de aislamiento deben tener una dimensión de 20$24 \mathrm{mt}^{2}$ y además de cumplir con las recomendaciones de estructura y equipamiento propias de un cupo UCI deben contar con la posibilidad de monitorización a distancia, con un área para lavamanos y cambio de delantales, lugar para guardar material limpio y sucio, con ventana de observación con persianas que se puedan limpiar (aseo concurrente y terminal) y con un sistema de presión positiva y negativa que permita que el 100\% del aire sea expulsado hacia fuera del recinto. e) Cupos para alojamiento conjunto: Las unidades Nivel III y IV deben tener disponibilidad de al menos un cupo que permita al cuidador pernoctar con el recién nacido previo al alta y al equipo de salud observar y evaluar la idoneidad del familiar responsable para un alta segura en recién nacidos de riesgo. Este cupo debe tener una superficie de al menos $20 \mathrm{mt}^{2}$ que asegure espacio suficiente para la cuna, la cama del cuidador, disponibilidad de baño con ducha y espacios para guardar enseres personales.

f) Cupo de procedimientos: Las unidades nivel III o IV podrán destinar un cupo de al menos $20 \mathrm{mt}^{2}$ para la realización de procedimientos quirúrgicos de urgencia. Este cupo de procedimientos debe contar con las características de un cupo de UCI en cuanto a infraestructura (iluminación, recambio de aire, etc.) y equipamiento y estar localizado en o adyacente al sector de cupos de intensivo.

g) El diseño de las unidades contempla espacios para la mantención de equipamiento como incubadoras de transporte, carros de paro, ecógrafos, equipos de radiología portátil y otros.

Todos los establecimientos deben contar con al menos una incubadora de transporte básica para traslados de baja complejidad internos o entre centros. Los requerimientos son de una incubadora en unidades con menos de 30 cupo y 2 incubadoras en unidades de más de 30 cupos. Los Centros Neonatales de Nivel II, III y IV deben contar con incubadora y ventilador mecánico de transporte, bombas de infusión de jeringa y monitor con ECG, saturometría, presión no invasiva e invasiva, todos con autonomía mínima de 2 horas. Este requerimiento se puede eliminar para centros Nivel II si existe convenido un sistema de rescate desde el centro neonatal nivel III o IV asignado para la red.

\section{Criterios de ingreso según nivel de complejidad}

La organización de la atención neonatal en niveles de complejidad concordantes con los niveles de complejidad de los servicios de ginecología y obstetricia tiene como objetivo que las embarazadas y los recién nacidos reciban atención en centros con capacidades resolutivas acordes a los requerimientos de la condición materna y neonatal: El modelo de organización de la atención neonatal tiene entre sus objetivos prevenir el traslado neonatal privilegiando el traslado materno cuando por riesgo materno o fetal corresponda.

Tabla 2 muestra los criterios de ingreso según nivel de complejidad. 

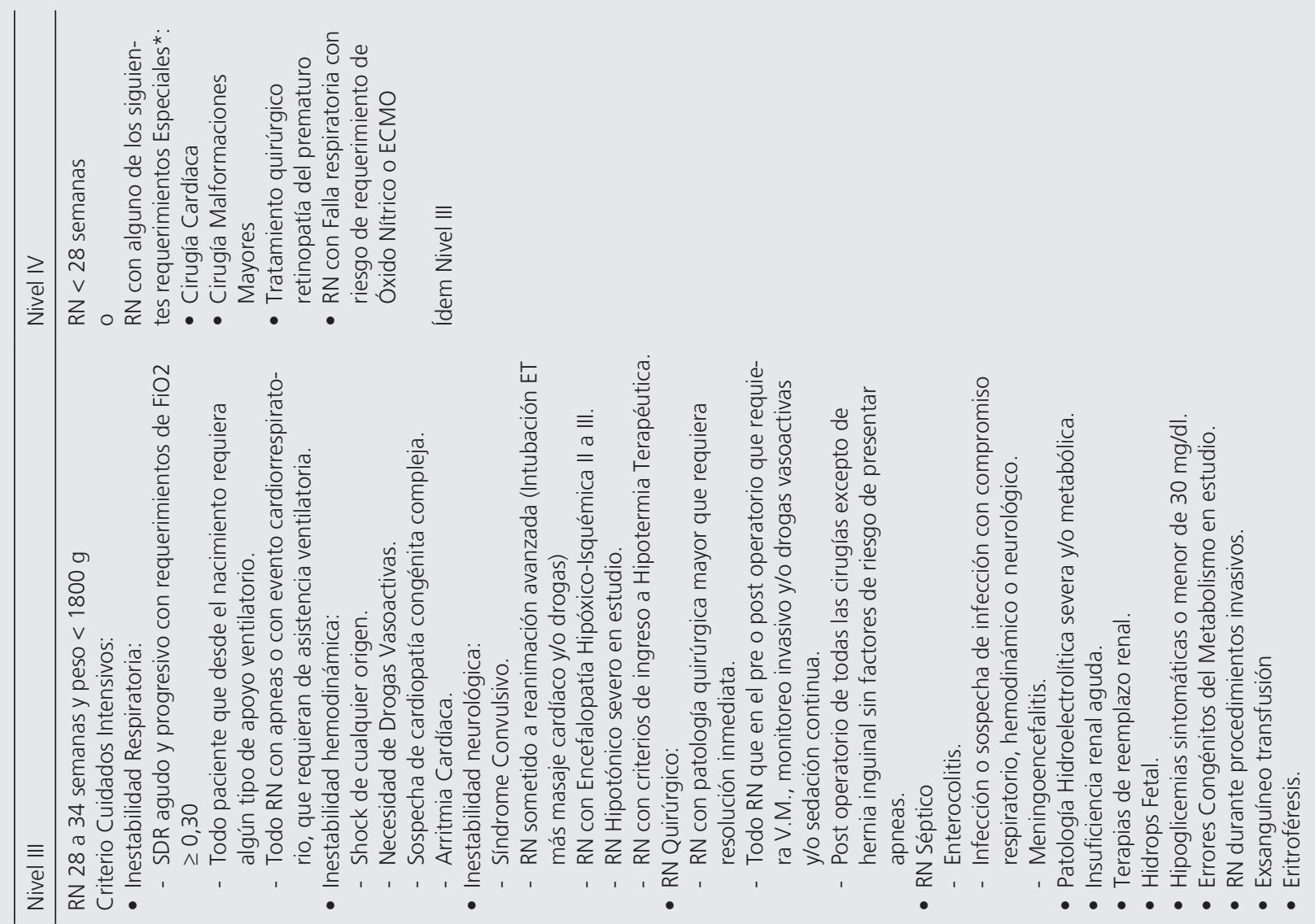

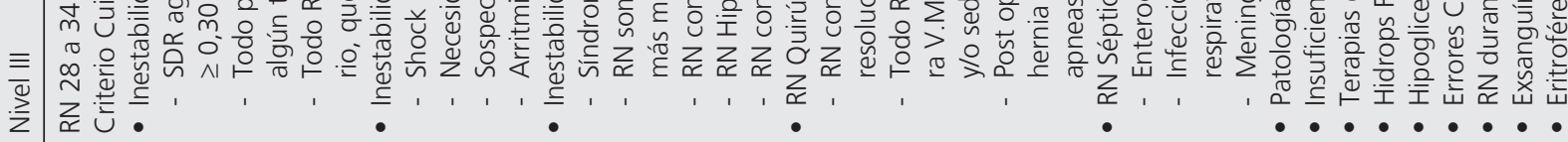

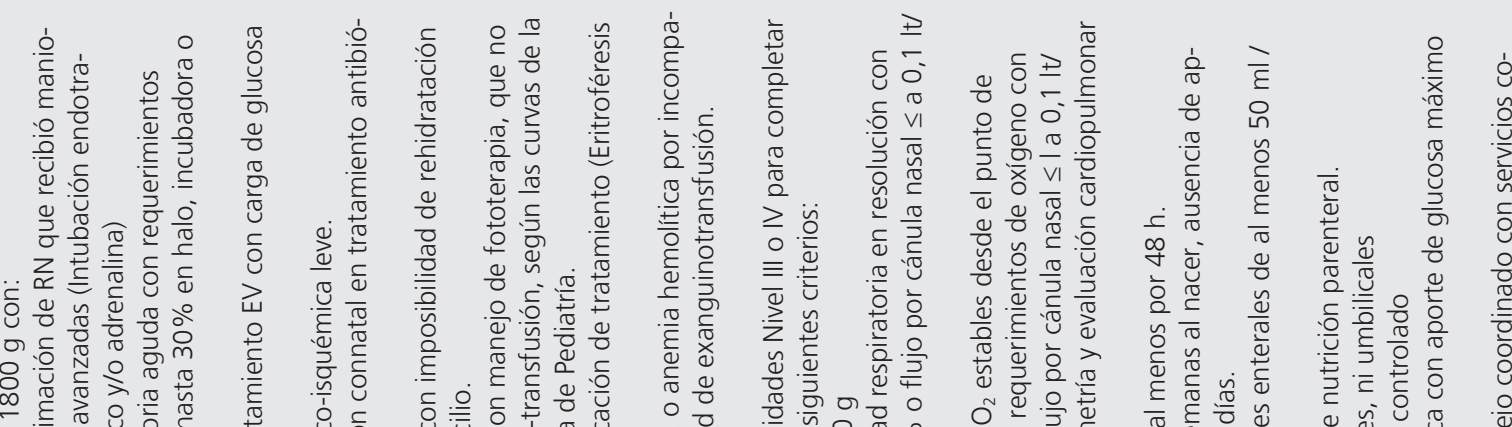

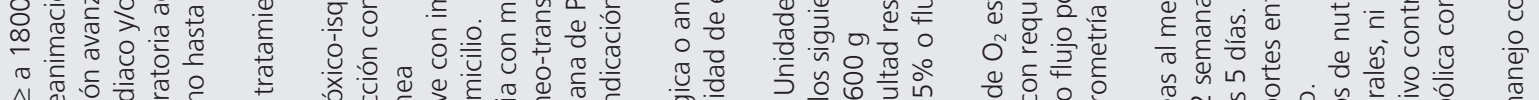

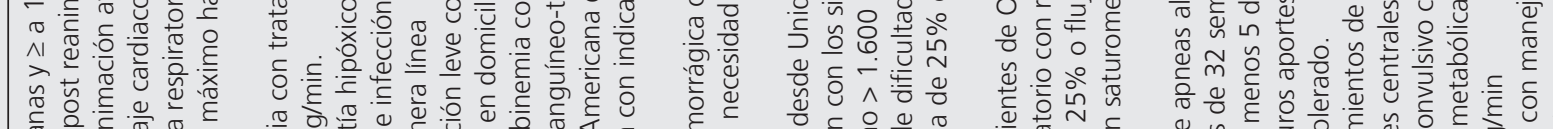

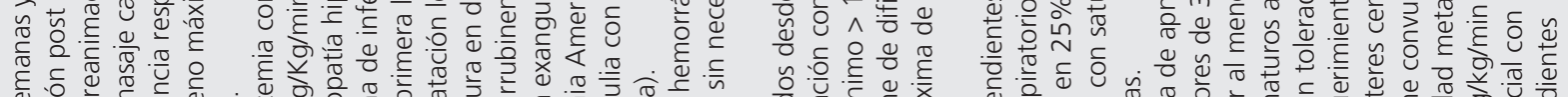

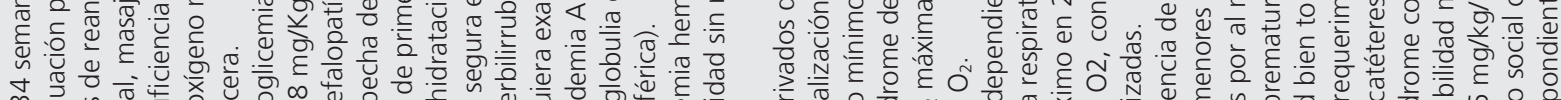

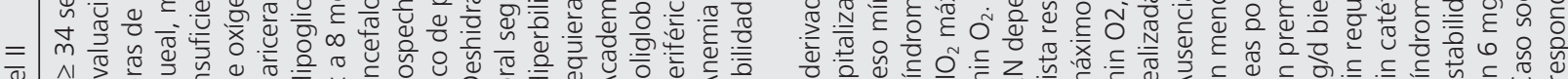

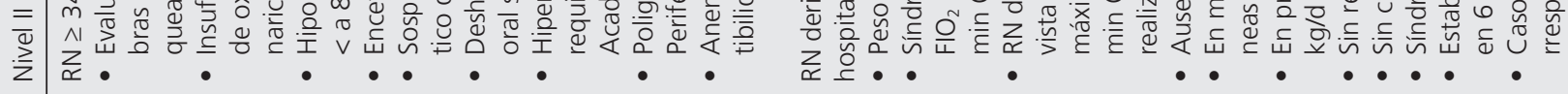

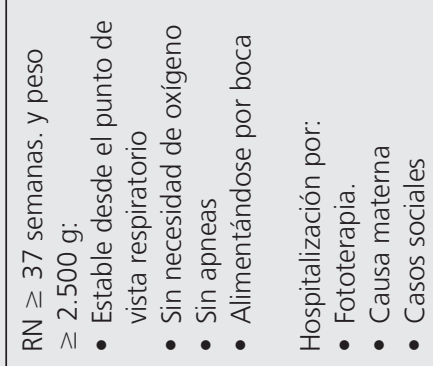




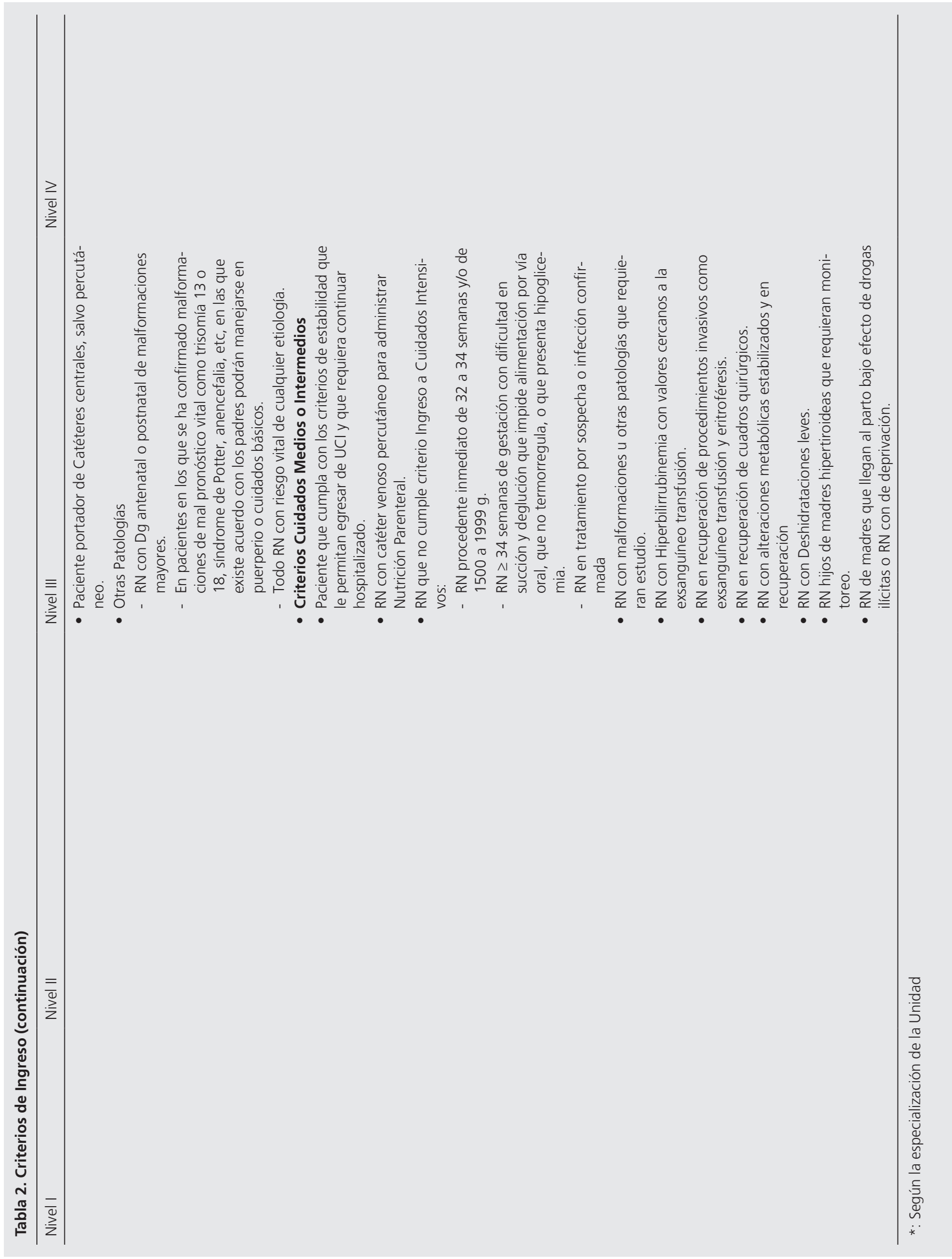




\section{Otras actividades asistenciales y no asistencia- les de las unidades neonatales}

En cada nivel de complejidad se realizan distintas actividades que no corresponden a actividades asistenciales directas con el neonato hospitalizado o con su atención ambulatoria, pero que forman parte de las actividades necesarias para la gestión de la unidad y para dar cumplimiento a los distintos requerimientos que la normativa vigente determina.

La dotación de recursos humanos de cada unidad debe planificarse tomando en consideración las cargas asistenciales directas recomendadas en la tabla 1 y las actividades que deben ejecutarse en las unidades según su nivel de complejidad, y que requieren la presencia o participación de los profesionales y técnicos de la unidad. Algunas de ellas corresponden a actividades asistenciales ambulatorias, de coordinación con los otros niveles de complejidad de la red y con los equipos obstétricos; otras corresponden a actividades no asistenciales relacionadas con la capacitación interna de los equipos de profesionales y técnicos, educación a los padres y otras necesarias para la gestión. La tabla 3 muestra algunas de estas actividades según cada nivel de complejidad y que requieren la participación de médicos, enfermeras o matronas y/o técnicos de la unidad.

Tabla 3. Otras Actividades Asistenciales y no Asistenciales de la Unidades Neonatales

\begin{tabular}{|c|c|c|c|}
\hline & Nivel I & Nivel II & Nivel III y IV \\
\hline Médicos & $\begin{array}{l}\text { - Reuniones clínicas y de capacita- } \\
\text { ción interna }{ }^{1} \\
\text { - Reuniones administrativas }{ }^{1}\end{array}$ & $\begin{array}{l}\text { - } \text { Comité de Morbimortalidad² } \\
\text { - Interconsultas centros de la red² } \\
\text { - Reuniones clínicas y de capacita- } \\
\text { ción interna }{ }^{1}\end{array}$ & $\begin{array}{l}\text { - Participación en Comités de: } \\
\text { - Morbimortalidad² } \\
\text { - Comité de Perinatología }{ }^{2} \\
\text { - Comité de IAAS } \\
\text { - Comité de lactancia materna } \\
\text { - Comité de Calidad } \\
\text { - Auditoría de partos prematuros } \\
\text { - Atención ambulatoria Seguimiento de Prema- } \\
\text { turos } \\
\text { - Interconsultas centros de la red² } \\
\text { - Coordinación traslado pacientes }{ }^{2} \\
\text { - Reuniones clínicas y de capacitación interna } \\
\text { - Reuniones administrativas }\end{array}$ \\
\hline $\begin{array}{l}\text { Enfermeras/ } \\
\text { Matronas }\end{array}$ & $\begin{array}{l}\text { - Reuniones clínicas y de capacita- } \\
\text { ción interna }{ }^{1} \\
\text { - Reuniones administrativas }{ }^{1} \\
\text { - } \text { Actividades de educación a los } \\
\text { padres }^{3} \\
\text { - } \text { Registros de datos neonatales }^{4}\end{array}$ & $\begin{array}{l}\text { - Reuniones clínicas y de capacita- } \\
\text { ción interna }{ }^{1} \\
\text { - Reuniones administrativas }{ }^{1} \\
\text { - } \text { Actividades de educación a los } \\
\text { padres }^{3} \\
\text { - }{\text { Registros de datos neonatales }{ }^{4}}\end{array}$ & $\begin{array}{l}\text { - Reuniones clínicas y de capacitación interna² } \\
\text { - Participación en Comités de: } \\
\text { - Morbimortalidad }{ }^{2} \\
\text { - Comité de Perinatología }{ }^{2} \\
\text { - Comité de IAAS } \\
\text { - Comité de lactancia materna } \\
\text { - Comité de Calidad } \\
\text { - Auditoría de partos prematuros } \\
\text { - Reuniones administrativas }{ }^{2} \\
\text { - Actividades de educación a los padres }{ }^{3} \\
\text { - Registros y envío de datos neonatales }{ }^{4}\end{array}$ \\
\hline $\begin{array}{l}\text { Técnicos } \\
\text { paramédicos }\end{array}$ & $\begin{array}{l}\text { - Reuniones clínicas y de capacita- } \\
\text { ción interna }{ }^{2} \\
\text { - Reuniones administrativas }{ }^{2}\end{array}$ & $\begin{array}{l}\text { - Reuniones clínicas y de capacita- } \\
\text { ción interna } \\
\text { - Reuniones administrativas }{ }^{2} \\
\text { - } \text { Actividades de educación a los } \\
\text { padres }^{3}\end{array}$ & $\begin{array}{l}\text { - Reuniones clínicas y de capacitación interna² } \\
\text { - Reuniones administrativas }{ }^{2} \\
\text { - Actividades de educación a los padres }\end{array}$ \\
\hline
\end{tabular}

${ }^{1}$ Estas actividades requieren la participación de todos los médicos de la unidad y en su conjunto la cada unidad debe estimar al menos $2 \mathrm{~h}$ semanales a estas actividades. ${ }^{2}$ Estas actividades son asignadas a un médico de la unidad según un esquema de rotación que cada unidad determina. En conjunto las unidades Nivel III y IV deben asignar un mínimo de $11 \mathrm{~h}$ semanales, asegurando al menos $3 \mathrm{~h}$ a Seguimiento de Prematuros, $2 \mathrm{~h}$ a participación en Comité de Perinatología e interconsultas a alto riesgo obstétrico y $6 \mathrm{~h}$ a interconsultas a centros de red, coordinación de traslados y colaboración en gestión clínica de casos complejos. ${ }^{3}$ Esta actividad se asigna a una matrona/enfermera y técnico según esquema de rotación propio de cada unidad. Para unidades de nivel III y IV debe considerarse al menos $4 \mathrm{~h}$ semanales a esta actividad. En las unidades Nivel I y II, estas actividades se pueden realizar en conjunto con unidad de pediatría y debe considerarse al menos $2 \mathrm{~h}$ de asignación semanal. "Esta actividad se asigna a una matrona o enfermera según esquema propio de la unidad y en su conjunto se debe estimar al menos $4 \mathrm{~h}$ semanales para el caso de las unidades nivel III y IV. En las unidades Nivel I y II estas actividades se pueden realizar en conjunto con unidad de pediatría y debe considerarse al menos 2 h de asignación semanal. RN: Recién Nacido; ECMO: Oxigenación por Membrana Extracorpórea; VM: Ventilación Mecánica; IAAS: Infecciones Asociadas a la Atención de Salud; ET: Endotraqueal; SDR: Síndrome de Dificultad Respiratoria. 


\section{Procedimientos quirúrgicos de urgencia en unidad de neonatología}

El traslado y estadía en pabellón del recién nacido quirúrgico sea asocia a diversas complicaciones como hipotermia, deterioro hemodinámico, alteraciones ventilatorias y de la oxigenación, desplazamiento o retiro de dispositivos de soporte vital, etc. por esta razón, en casos de pacientes en condición de extrema gravedad con indicación de cirugía (tabla 4), la ejecución de procedimientos quirúrgicos en las unidades de Neonatología Nivel III o IV es la alternativa recomendada. Los cupos UCI pueden ser utilizados para este fin, ya que cumplen con las condiciones de seguridad y con las características de infraestructura (temperatura ambiental, renovación de aire, iluminación, accesos eléctricos, etc.) y equipamiento necesario para la ejecución de procedimientos de urgencia en paciente grave

Las unidades actualmente en funcionamiento $\mathrm{cu}-$ yos cupos de cuidados intensivos no cumplen con estas condiciones para poder realizar procedimientos invasivos, deben habilitar una sala de procedimientos de al menos 16 a $20 \mathrm{mt}^{2}$ que cumpla con las recomendaciones de infraestructura y equipamiento arriba mencionados.

La ejecución de procedimientos quirúrgicos en la unidad de neonatología requiere:

- Encargado de la preparación del espacio físico y equipamiento neonatal para el procedimiento: Aseo, distribución de equipamiento, señalización de procedimiento en curso, etc.

- Enfermera / Matrona encargada de preparar al paciente para el procedimiento y de velar por que este se desarrolle de la forma segura colaborando con el equipo quirúrgico en el cuidado del paciente durante la cirugía y en la etapa de estabilización posterior
Tabla 4. Condiciones clínicas y patologías que pueden requerir cirugía en la UCIN

- RN prematuros < $1.500 \mathrm{~g}$ con indicación quirúrgica

- RNT con inestabilidad hemodinámica con indicación quirúrgica

- Paciente en ventilación de alta frecuencia u óxido nítrico inhalado previo a cirugía

- Cierre ductal en $<2.000 \mathrm{~g}$ o en paciente inestable

- Laparotomía para Enterocolitis necrotizante, perforación intestinal, malrotación, obstrucción intestinal

- Reducción o reparación de defectos de la pared abdominal

- Hernia diafragmática inestable

- Canulación o decanulación para ECMO

- Cualquier cirugía o procedimiento en paciente gravemente enfermo

RN: Recién Nacido; RNT: Recién Nacido de Término; ECMO: Oxigenación por Membrana Extracorpórea.

- Técnico paramédico asignado al procedimiento para apoyo del equipo neonatal y quirúrgico.

- Neonatólogo asignado al paciente durante el procedimiento quirúrgico y la estabilización posterior.

- Protocolo local de cómo realizar un procedimiento quirúrgico (condiciones, prevención IASS, restricciones de acceso, etc.)

El equipo quirúrgico y de pabellón debe trasladarse a la unidad con todo el personal y equipamiento necesario y es el responsable del procedimiento quirúrgico, propiamente tal, del proceso anestésico y del manejo del paciente mientras dure la cirugía. Es también responsable de que se cumplan los protocolos de calidad y seguridad y de la solicitud de consentimiento informado a los familiares del paciente.

\section{Conflicto de intereses}

Los autores declaran no tener conflicto de intereses.

\section{Referencias}

1. Papile LA, Baley JE, Benitz W, et al. Levels of neonatal care. Pediatrics. 2012;130(3):587-97.

2. Brown BB, Patel C, McInnes E, Mays N, Young J, Haines M. The effectiveness of clinical networks in improving quality of care and patient outcomes: a systematic review of quantitative and qualitative studies. BMC Health Serv Res. 2016;16:360. Published 2016 Aug 8. doi:10.1186/s12913-016-1615-z

3. Kilpatrick SJ, Menard MK, Zahn CM, Callaghan WM. Levels of Maternal Care: Obstetric Care Consensus No, 9. Obstet Gynecol. 2019 08;134(2):e41-e55.

4. Walther F, Küster DB, Bieber A, et al.
Impact of regionalisation and casevolume on neonatal and perinatal mortality: an umbrella review. BMJ Open. 202009 25;10(9):e037135.

5. Cifuentes J, Bronstein J, Phibbs CS, Phibbs RH, Schmitt SK, Carlo WA. Mortality in low-birthweight infants according to level of neonatal care at hospital of birth. Pediatrics. 2002;109(5):745-51.

6. Chung JH, Phibbs CS, Boscardin WJ, Kominski GF, Ortega AN, Needleman $J$. The effect of neonatal intensive care level and hospital volume on mortality of very low birth weight infants. Med Care. 2010;48(7):635-44.

7. Lasswell SM, Barfield WD, Rochat RW, Blackmon L. Perinatal regionalization for very low-birthweight and very preterm infants: a meta-analysis. JAMA. 2010;304(9):992-1000.

8. Binder S, Hill K, Meinzen-Derr J, Greenberg JM, Narendran V. Increasing VLBW deliveries at subspecialty perinatal centers via perinatal outreach. Pediatrics. 2011;127(3):487-93

9. Gale C, Santhakumaran S, Nagarajan S, et al. Impact of managed clinical networks on NHS specialist neonatal services in England: population-based study. BMJ. 2012;344:e2105.

10. Watson SI, Arulampalam W, Petrou $S$, et al. The effects of designation and volume of neonatal care on mortality and morbidity outcomes of very preterm infants in England: retrospective 
population-based cohort study. BMJ Open. 2014;4(7):e004856.

11. Jensen EA, Lorch SA. Effects of a Birth Hospital's Neonatal Intensive Care Unit Level and Annual Volume of Very Low-Birth-Weight Infant Deliveries on Morbidity and Mortality. JAMA Pediatr. 2015;169(8):e151906.

12. Snowden JM, Cheng YW, Emeis CL, Caughey AB. The impact of hospital obstetric volume on maternal outcomes in term, non-low-birthweight pregnancies. Am J Obstet Gynecol. 2012;212(3):380. e1-9.

13. Kozhimannil KB, Thao V, Hung P, Tilden E, Caughey AB, Snowden JM. Association between Hospital Birth Volume and Maternal Morbidity among Low-Risk Pregnancies in Rural, Urban, and Teaching Hospitals in the United States. Am J Perinatol. 2016;33(6):590-9.

14. Hehir MP, Ananth CV, Wright JD, Siddiq Z, D'Alton ME, Friedman AM. Severe maternal morbidity and comorbid risk in hospitals performing $<1000$ deliveries per year. Am J Obstet Gynecol. 2017;216(2):179.e1-179.e12.

15. Desplanches T, Blondel B, Morgan AS, et al. Volume of Neonatal Care and Survival without Disability at 2 Years in Very Preterm Infants: Results of a French National Cohort Study. J Pediatr. 2019;213:22-29.e4.

16. Hentschel R, Guenther K, Vach W, Bruder I. Risk-adjusted mortality of VLBW infants in high-volume versus low-volume NICUs. Arch Dis Child Fetal Neonatal Ed. 2019;104(4):F390-F395.

17. Ismail AQT, Boyle EM, Pillay T, et al. The impact of level of neonatal care provision on outcomes for preterm babies born between 27 and 31 weeks of gestation, or with a birth weight between 1000 and $1500 \mathrm{~g}$ : a review of the literature. BMJ Paediatr Open. 2020;4(1):e000583.

18. Goodarzi B, Walker A, Holten L, et al. Towards a better understanding of risk selection in maternal and newborn care: A systematic scoping review. PLoS One. 2020;15(6):e0234252.

19. Helenius K, Longford N, Lehtonen L, Modi N, Gale C. Association of early postnatal transfer and birth outside a tertiary hospital with mortality and severe brain injury in extremely preterm infants: observational cohort study with propensity score matching. BMJ. 2019;367: 15678 .
20. Marlow N, Bennett C, Draper ES, Hennessy EM, Morgan AS, Costeloe KL. Perinatal outcomes for extremely preterm babies in relation to place of birth in England: The EPICure 2 study. Arch Dis Child Fetal Neonatal Ed. 2014;99(3):F1818.

21. Rautava L, Lehtonen L, Peltola M, et al. The effect of birth in secondary- or tertiary-level hospitals in Finland on mortality in very preterm infants: a birth-register study. Pediatrics. 2007;119(1):e257-63.

22. Helenius K, Gissler M, Lehtonen L. Trends in centralization of very preterm deliveries and neonatal survival in Finland in 1987-2017. Transl Pediatr. 2019;8(3):227-32.

23. Rite Gracia S, Fernández Lorenzo JR, Echániz Urcelay I, et al. [Health care levels and minimum recommendations for neonatal care]. An Pediatr (Barc). 2013;79(1):51.e1-51.e11.

24. Optimal Arrangements for Neonatal Intensive Care Units in the UK (2014) A BAPM Framework for Practice [Internet]. www.bapm.org. 2014 [citado 13 diciembre 2020]. Disponible en: https:// www.bapm.org/resources/31-optimalarrangements-for-neonatal-intensivecare-units-in-the-uk-2014.

25. Unidades de Neonatología Estándares y recomendaciones de calidad [Internet]. https://www.mscbs.gob. es/. 2014 [citado 13 diciembre 2020]. Disponible en: https://www.mscbs.gob.es/ organizacion/sns/planCalidadSNS/docs/ NEONATOLOGIA_Accesible.pdf

26. Safe, sustainable and productive staffing an improvement resource for neonatal care [Internet]. 1. ${ }^{\mathrm{a}}$ ed. London: National Quality Board (NQB).; 2018 [citado 13 diciembre 2020]. Disponible en: https:// improvement.nhs.uk/documents/2978/ Safe_Staffing_Neonatal_FINAL_ PROOF_27_June_2018.pdf

27. Novoa P José M, Milad A Marcela, Vivanco G Guillermo, Fabres B Jorge, Ramírez F Rodrigo. Recomendaciones de organización, características y funcionamiento en Servicios o Unidades de Neonatología. Rev. chil. pediatr. [Internet] 2009 [citado 2020 Dic 14];80(2):168-87

28. Leyton B, Becerra C, Castillo C, Moya C, Strain H, Santander S. Norma Administrativa Programa Nacional de Salud de la Infancia [Internet]. 1. ${ }^{\mathrm{a}}$ ed.
Santiago: Ministerio de Salud; 2013 [citado 13 diciembre 2020]. Disponible en: http://www.repositoriodigital. minsal.cl/bitstream/handle/2015/437/ Norma-Administrativa-ProgramaNacional-de-Salud-de-la-Infancia-2013. pdf? sequence $=1$ \&isAllowed $=y$

29. Manual de procedimientos para la atención del recién nacido en el período inmediato y puerperio en servicios de obstetricia y ginecología [Internet]. 1. ${ }^{\mathrm{a}}$ ed. Santiago: Ministerio de Salud Chile; 2014 [citado 13 diciembre 2020]. Disponible en: https://www. minsal.cl/sites/default/files/files/ MANUALDEPROCEDIMIENTOSRN 10112014.pdf

30. Orientaciones para la planificación y programación en red 2020 [Internet]. Santiago: Ministerio de Salud Chile; 2020 [citado 13 diciembre 2020]. Disponible en: https://www.minsal.cl/ wp-content/uploads/2019/09/2019.09.09_ ORIENTACIONES-PARA-LAPLANIFICACION-EN-RED-2020_v3.pdf

31. Neonatal units: planning and design (HBN 09-03) Guidance for the design of hospital neonatal units including special care, family spaces and specialist clinical rooms. [Internet]. Department of Health and Social Care; 2003 [citado el 13 de diciembre de 2020]. Disponible en: https://assets.publishing.service.gov. uk/government/uploads/system/uploads/ attachment_data/file/147879/HBN_0903_Final.pdf

32. Hignett S, Lu J, Fray M. Two Case Studies Using Mock-Ups for Planning Adult and Neonatal Intensive Care Facilities. J Healthc Eng 2010;1(3) 399413.

33. White RD, Smith JA, Shepley MM, et al. Recommended standards for newborn ICU design, eighth edition. J Perinatol. 2013;33 Suppl 1:S2-16.

34. EFCNI, Moen A, Hallberg B et al., European Standards of Care for Newborn Health: General layout of the unit. 2018. https://newborn-health-standards.org/ wp-content/uploads/2019/04/TEG_ NICU-design_complete.pdf

35. O'Callaghan N, Dee A, Philip RK. Evidence-based design for neonatal units: a systematic review. Matern Health Neonatol Perinatol. 2019; 5:6.

36. White RD. Recommended standards for newborn ICU design, 9th edition. J Perinatol. 2020;40(Suppl 1):2-4. 